\title{
Tendência de Consumo de Alimentos Industrializados por Portadores de Doenças Crônicas Não Transmissíveis
}

\author{
Cristina Henschel Matos ${ }^{1}$, Franciele Aparecida de Souza da Cruz $^{2}$, \\ Joyce Aparecida de Oliveira Pereira ${ }^{3}$, Rosana Henn ${ }^{4}$
}

\begin{abstract}
RESUMO
As Doenças Crônicas Não Transmissíveis (DCNTs) são hoje uma das principais razões de óbitos no Brasil e no mundo, tendo a má alimentação como um dos fatores determinantes. Dessa forma, este estudo visou a avaliar o conhecimento e a tendência de consumo de alimentos industrializados por usuários portadores de DCNTs de uma Unidade de Saúde Escola de Itajaí-SC. A população foi constituída por mulheres adultas, portadoras de DCNT, assistidas pela Unidade de Saúde entre os meses de setembro/2017 e março/2018. Os dados foram coletados por meio de entrevista contendo questões sobre fatores que influenciam a compra e o consumo de alimentos, rotulagem e dificuldades de interpretação das informações das embalagens. Os resultados demonstraram que das 59 mulheres avaliadas a maioria era adulta de meia idade, com Ensino Fundamental incompleto e renda per capta inferior a um salário mínimo. Dentre as DCNTs mais relatadas estavam a hipertensão arterial sistêmica a diabetes mellitus. Destaca-se que o preço foi fator preponderante para a aquisição dos alimentos (56\%), bem como o fato de os mesmos estarem em promoção (54\%). Observou-se, também, que existe uma tendência de compra para os produtos ditos mais saudáveis, entretanto somente $61 \%$ das mulheres têm o hábito de observar a composição nutricional dos alimentos e, destas, 33\% apresentam dificuldades na sua interpretação. Diante deste contexto, é possível verificar a necessidade de atividades de educação alimentar e nutricional que levem à reflexão sobre o ato de se alimentar, buscando a desassociação entre alimento industrializado e preço com alimentos saudáveis.
\end{abstract}

Palavras-chave: Doença crônica. Alimentos industrializados. Rotulagem de alimentos.

CONSUMPTION TENDENCY OF PROCESSED FOODS BY CARRIES OF NON-COMMUNICABLE CHRONIC DISEASES

\section{ABSTRACT}

Non-communicable chronic diseases (CDN) are today one of the main causes of death in Brazil and in the world, with poor diet being one of the determining factors Thus, the study aimed to evaluate the knowledge and tendency of consumption of industrialized foods by users with CDNT from a School Health Unit of Itajaí-SC. The population was made up of adult women with CNCDs, assisted by the Health Unit between September and December 2017. Data was collected through an interview containing questions about factors that influence the purchase and consumption of food, labeling and difficulties in interpreting packaging information. The results showed that of the 59 women most evaluated were middle-aged adults with incomplete primary education and per capita incomes below minimum wage, among the most commonly reported NCDs were Systemic Arterial Hypertension and Diabetes Mellitus. It should be emphasized that price was a preponderant factor for the acquisition of food (56\%), as well as the fact that they were in promotion (54\%). It was also observed that there is a tendency to buy healthier products, therefore, only $61 \%$ of women have a habit of observing the nutritional value of food, and of these, $33 \%$ presents difficulties in their interpretation. Given this context, it is possible to verify the need for food and nutritional education activities which leads to reflection about the act of eating, seeking the disassociation between industrialized food and the price of healthy food.

Keywords: Chronic disease. Industrialized foods. Food labeling.

RECEBIDO EM: 14/9/2018

MODIFICAÇÕES REQUERIDAS EM: 19/7/2019

ACEITO EM: 8/8/2019

\footnotetext{
Graduação em Nutrição (1997) e Mestrado em Engenharia de Produção (2000) pela Universidade Federal de Santa Catarina (UFSC). Especialização em Fitoterapia aplicada à nutrição pela AVM Faculdades Integradas (2016). Professora titular da Universidade do Vale do Itajaí (Univali). Tem experiência na área de Nutrição, com ênfase em Análise Nutricional de População, atuando principalmente nos seguintes temas: estado nutricional, educação alimentar e nutricional, comportamento alimentar, obesidade, anemia ferropriva e doenças do trato digestório. matoscris@gmail.com

2 Acadêmica do Curso de Nutrição - Universidade do Vale do Itajaí - Univali. fran.souzacruz@hotmail.com

Acadêmica do Curso de Nutrição - Universidade do Vale do Itajaí - Univali. joyce.010@hotmail.com

${ }^{4}$ Mestre em Ciência dos Alimentos - Universidade Federal de Santa Catarina - UFSC. Professora do Curso de Nutrição - Universidade do Vale do Itajaí - Univali. rhenn@univali.br
} 


\section{INTRODUÇÃO}

As Doenças Crônicas Não Transmissíveis (DCNTs) são, hoje, uma das principais causas de óbitos no Brasil e no mundo. Segundo dados da Organização das Nações Unidas (ONU), cerca de 43 milhões de pessoas morrem todos os anos em decorrência dessas enfermidades; isso corresponde à, aproximadamente, $80 \%$ das causas de mortes no mundo. Entre as principais estão doenças cardíacas, acidente vascular cerebral, câncer, diabetes mellitus e doenças respiratórias (BRASIL, 2015).

De acordo com a Pesquisa Nacional da Saúde (PNS), cerca de $40 \%$ da população adulta, o equivalente a 57,4 milhões de pessoas, possuíam pelo menos uma DCNT em 2014, sendo em 2013 a causa de aproximadamente $72,6 \%$ das mortes no país (BRASIL, 2014a).

Essas enfermidades acometeram grande parte da população nos últimos anos, afetando diretamente a qualidade de vida em razão de suas limitações e grandes custos econômicos (MALTA, 2014). As DCNTs desenvolvem-se durante todo o ciclo de vida em razão de vários fatores e por longos períodos (BRASIL, 2014a).

Dentre as causas que contribuem para o desenvolvimento das DCNTs, a Organização Mundial da Saúde pontua os quatro principais fatores, sendo eles o fumo, o sedentarismo, o uso prejudicial de álcool e a má alimentação (DUNCAN, 2012).

Diante do cenário de saúde preocupante do Brasil, o Ministério da Saúde atualizou o Guia Alimentar Para a População Brasileira, visando a conscientizar a sociedade para que adotem hábitos alimentares mais saudáveis. Este guia classifica os alimentos em quatro categorias: alimentos in natura, minimamente processados, processados e ultraprocessados, além de orientar a frequência de consumo de cada um (BRASIL, 2014b).

O Guia traz como alimentos in natura os de origem vegetal ou animal que não sofreram nenhum tipo de alteração após saírem da natureza. Os minimamente processados são os alimentos que passam por pequenos processos preservando-os e tornando-os apropriados para armazenamento, como limpeza, remoção de partes não comestíveis e refrigeração. Já os alimentos processados são aqueles fabricados pela indústria utilizando alimentos in natura, porém com a adição de sal, açúcar ou outra substância de uso culinário para melhorar as propriedades organolépticas ou aumentar sua durabilidade (BRASIL, 2014b).
Por fim, alimentos ultraprocessados são considerados formulações criadas pela indústria envolvendo diversas etapas e técnicas de processamento e muitos ingredientes, incluindo sal, açúcar, óleos e gorduras e substâncias de uso exclusivamente industrial, e, quando existentes, os alimentos in natura ou minimamente processados apresentam uma concentração inferior em relação aos ingredientes ultraprocessados (MARTINS, 2013).

Assim sendo, alimentos ultraprocessados possuem uma quantidade maior de calorias, além de conter mais açúcar e menos fibras quando comparados aos alimentos in natura ou minimamente processados. Não há estudos que comprovem o benefício deste tipo de alimento à qualidade nutricional da dieta (MONTEIRO et al., 2011; MARTINS, 2013). Em contrapartida, são explorados pelo comércio e indústria alimentícia, que os tornam mais acessíveis para todas as faixas etárias, oferecendo alimentos cada vez mais práticos, palatáveis, duráveis e atrativos para toda a população (MONTEIRO et al., 2010; BIELEMANN et al., 2015).

Em virtude das mudanças nos ingredientes utilizados na elaboração dos alimentos industrializados, a embalagem e a rotulagem dos alimentos são de grande importância para que seja estabelecida uma conexão entre o produto e o consumidor. Os rótulos servem para informar sobre o que está sendo ingerindo, porém, pela falta de conhecimento ou dificuldade de entendê-los, os consumidores acabam não dando a devida importância na hora da compra e, consequentemente, fazendo escolhas que não contribuem para uma alimentação saudável (BENDINO; POPOLIM; OLIVEIRA, 2012).

Um estudo realizado por Santos et al. (2016) verificou associação entre a compra de alimentos industrializados com a alta taxa de prevalência de DCNTs, relacionadas ao frágil conhecimento em educação e saúde da população em geral e também à interpretação de rótulos de alimentos.

Diante do exposto, o objetivo deste estudo foi avaliar o conhecimento e tendência de consumo de alimentos industrializados por pacientes com doenças crônicas não transmissíveis usuários do sistema único de saúde.

\section{METODOLOGIA}

Este artigo é um estudo de abordagem transversal, quantitativo, exploratório e descritivo. A população foi constituída por usuários assistidos por uma Unidade de Saúde Escola - USE - do município de 
Itajaí-SC, entre setembro/2017 e março/2018. Os indivíduos foram selecionados por demanda espontânea e de acordo com os seguintes critérios de inclusão: adultos (20 a 59 anos), sexo feminino e portador de, ao menos, uma das seguintes DCNTs registradas em prontuário: hipertensão arterial sistêmica, diabetes mellitus, dislipidemia e obesidade. O projeto foi submetido ao Comitê de Ética em Pesquisa da Univali e aprovado sob protocolo 2.267.109 de 11 de setembro de 2017. A participação foi voluntária e expressa por meio da assinatura do Termo de Consentimento Livre e Esclarecido.

A coleta de dados foi realizada pelas acadêmicas responsáveis pelo projeto em local calmo e tranquilo. A entrevista abrangeu a identificação e a seleção dos usuários atendidos na USE que aguardavam consultas nas especialidades, abordados e esclarecidos sobre a possível participação na pesquisa. Na sequência foram realizadas algumas perguntas a fim de verificar o enquadramento nos critérios de inclusão.

A avaliação das características socioeconômicas foi feita com auxílio de um questionário semiestruturado na forma de entrevista, contendo questões referentes à identificação (sexo, idade) e aos dados socioeconômicos (renda e escolaridade). A avaliação das tendências de consumo de alimentos industrializados foi identificada mediante um questionário aplicado em forma de entrevista, contendo questões semiabertas sobre fatores que influenciam a compra dos alimentos, conhecimento e hábito de leitura dos rótulos e embalagens e dificuldade de interpretação das informações contidas nos rótulos.

Os dados coletados na pesquisa foram analisados com auxílio dos programas Microsoft Excel $^{\circ} \mathrm{e}$ Word $^{\circ}$, analisados e comparados com legislação e literatura recente sobre o tema. As variáveis quantitativas contínuas foram expressas por meio de médias e desvio-padrão e as variáveis categóricas por intermédio de frequências absolutas e relativas.

\section{RESULTADOS E DISCUSSÃO}

Foram avaliadas 59 mulheres com idade média de $48,86( \pm 14,1)$ anos. Quanto à escolaridade, a média foi de $7,27( \pm 4)$ anos de estudo, entretanto ressalta-se que $42 \%(n=25)$, relataram não ter o Ensino Fundamental completo. Verificou-se que a renda familiar média mensal foi de $\mathrm{R} \$ 2.368,25( \pm 1.479,84)$, havendo, em média, $3( \pm 1,3)$ pessoas por domicílio, obtendo-se, assim, o valor de renda mensal per capita de $\mathrm{R} \$ 755,05$.
Em um inquérito realizado pelo Vigitel no ano 2017, foram avaliados 1.005 adultos. Destes, 605 eram mulheres, observando-se uma média de 9 anos de estudo, dados semelhantes aos encontrados na presente pesquisa (DURANTE et al., 2017).

Em estudo realizado com 8.676 usuários de Unidades Básicas de Saúde (UBSs) de todas as regiões do Brasil, 2.014 entrevistados eram da Região Sul, sendo $75 \%$ ( $n=1.517)$ mulheres. Os autores constataram que $61,5 \%$ dos usuários da Região Sul foram classificados como classe $C$, já $28,1 \%$ como classe $A$ ou $B$ e outros $10,4 \%$ como classe D ou E. Destes usuários, $25,4 \%$ afirmaram receber algum tipo de auxílio governamental (GUIBU et al., 2017).

Destaca-se que a classe social pode ser um fator contribuinte para a aquisição de produtos. Lindemann, Oliveira e Mendoza-Sassi (2016) analisaram as dificuldades na obtenção de uma alimentação saudável de 1.264 usuários de unidades básicas de saúde do município de Pelotas, e verificaram que $57,6 \%$ dos entrevistados referiram que o custo dos alimentos saudáveis é elevado.

Um dos fatores de inclusão no estudo era ter o diagnóstico de, pelo menos, uma doença crônica não transmissível. Desta forma, as enfermidades mais citadas pelas mulheres avaliadas foram: hipertensão arterial sistêmica, diabetes mellitus, obesidade, dislipidemias, entre outras, posto que a mais prevalente foi a hipertensão arterial sistêmica em $69 \%(n=41)$ dos casos, seguida de diabetes mellitus em $25 \%(n=15)$.

Os resultados encontrados seguem a tendência de estudos populacionais, como o realizado por Andrade et al. (2015), que, a partir da Pesquisa Nacional da Saúde, avaliou 60.202 indivíduos com idade igual ou superior a 18 anos e verificou a prevalência de hipertensão arterial sistêmica de $21,4 \%$, sendo superior no sexo feminino, com $24,2 \%$. O aumento da prevalência de hipertensão arterial sistêmica pode ser atribuído ao crescimento da população mundial, ao envelhecimento populacional, além da exposição a comportamentos de risco, como maus hábitos alimentares, consumo de álcool e tabaco e exposição crônica ao estresse.

No que se refere aos dados de hábitos de compra, foi constatado que, na maioria das vezes, eram as mulheres entrevistadas que as realizavam (69\%; $\mathrm{n}=41$ ). Quando questionadas sobre o uso de listas de compras, $58 \%(n=34)$ declararam ter esta prática, considerando que $42 \%(n=25)$ afirmaram que suas listas eram referentes ao período de um mês e $97 \%(n=57)$ referiram ter sempre um valor máximo para realizar suas compras no supermercado. 
De acordo com Halles, Sokolowsky e Hilgemberg (2011), a educação financeira é refletida na administração do dinheiro, e o planejamento financeiro pessoal consiste em estabelecer e seguir uma estratégia mais ou menos deliberada e dirigida para a manutenção ou a acumulação de bens e valores que irão formar o patrimônio. Segundo os autores, o controle daquilo que se ganha e do que se gasta é fundamental para o equilíbrio financeiro; entretanto o desejo pelas marcas vistas, muitas vezes, como "Premium" pelos consumidores, está acima de seu poder aquisitivo. Uma pesquisa realizada por Carrazedo Marques da Costa Filho e De Mendonça Motta (2015), mostra que a preferência destas marcas por consumidores de baixa renda está relacionada à maior aceitação de suas famílias, pois, segundo a percepção dos mesmos, estas marcas, conhecidas como de "confiança", rendem mais.

Em contrapartida, a Tabela 1, ao apresentar os itens considerados importantes na compra de alimentos pelas mulheres entrevistadas, mostra que o preço ainda é o item de maior importância no momento da compra dos alimentos.

Tabela 1 - Itens considerados importantes na compra de alimentos industrializados. Itajaí, setembro/2017 a março/2018

\begin{tabular}{lcc}
\hline Itens que influenciam a compra & $\mathbf{n}$ & $\mathbf{\%}$ \\
\hline Sabor & 2 & 3 \\
Saúde & 21 & 35 \\
Praticidade & 0 & 0 \\
Preço & 33 & 56 \\
Marca & 3 & 6 \\
Outros & 0 & 0 \\
TOTAL & 59 & 100 \\
\hline
\end{tabular}

Fonte: Os autores, 2018.

Os resultados da Tabela 1 destacam, ainda, que, embora haja um grande interesse sobre o consumo de alimentos saudáveis $35 \%(n=21)$, o preço ainda é o fator preponderante na compra dos alimentos, citado por $56 \%(n=33)$ das entrevistadas.

Um estudo realizado em 2008 utilizando dados da Pesquisa de Orçamentos Familiares, demonstrou que a aquisição de uma alimentação seguindo as recomendações do Guia Alimentar da População Brasileira para uma família de quatro pessoas, geraria um aumento mensal nas despesas de $26,53 \%$. Tratando-se de uma família com baixo poder aquisitivo, isso, muitas vezes, torna-se inviável (BORGES et al., 2015).
Além dos fatores econômicos que influenciam muito nos hábitos de compras, outras fontes podem colaborar para a não aquisição de produtos mais saudáveis, como frutas e hortaliças. Figueira, Lopes e Modena (2016) avaliaram as barreiras do consumo de frutas e hortaliças, sendo declarado pelos entrevistados do estudo que, além do preço, outros fatores que contribuem para essa não aquisição são a qualidade e a acessibilidade. Para Oliveira et al. (2017), os aspectos culturais da população também podem estar relacionados às escolhas alimentares.

O Quadro 1 apresenta as situações que podem influenciar na aquisição dos alimentos, confirmando, mais uma vez, que as questões financeiras, discutidas anteriormente, ainda são as mais consideradas.

Quadro 1 - Situações que influenciaram na aquisição de um alimento. Itajaí, setembro/2017 a março/2018

\begin{tabular}{|l|c|c|c|c|}
\hline \multirow{2}{*}{ Item } & \multicolumn{2}{|c|}{ Sim } & \multicolumn{2}{|c|}{ Não } \\
\cline { 2 - 6 } & $\mathbf{n}$ & $\%$ & $\mathbf{n}$ & $\%$ \\
\hline $\begin{array}{l}\text { Já adquiriu um alimento, que não } \\
\text { precisava, pela embalagem. }\end{array}$ & 14 & 24 & 45 & 76 \\
\hline $\begin{array}{l}\text { Já adquiriu um alimento, que não } \\
\text { precisava, somente porque estava } \\
\text { na promoção. }\end{array}$ & 32 & 54 & 27 & 46 \\
\hline $\begin{array}{l}\text { Já adquiriu um alimento, que } \\
\text { não precisava, porque estava na } \\
\text { degustação. }\end{array}$ & & 32 & 40 & 68 \\
\hline $\begin{array}{l}\text { Já adquiriu um alimento, que } \\
\text { não precisava, porque estava no } \\
\text { expositor da fila. }\end{array}$ & 18 & 31 & 41 & 69 \\
\hline $\begin{array}{l}\text { Já adquiriu um alimento, que } \\
\text { não precisava, porque foi ao } \\
\text { supermercado com fome. }\end{array}$ & 23 & 39 & 36 & 61 \\
\hline
\end{tabular}

Fonte: Os autores, 2018.

A partir dos resultados do Quadro 1 pode-se verificar que $54 \%(n=32)$ das mulheres entrevistadas afirmaram já ter adquirido um alimento que não precisavam por estar em promoção. Observou-se, também, que $24 \%(n=14)$ adquiriram um alimento apenas por sentir-se atraído pela embalagem.

A forma da apresentação do produto vem ganhando cada vez mais espaço no mercado como uma maneira de chamar a atenção do cliente. Um estudo realizado por Ricci (2016) teve como objetivo identificar as diferentes formas de apresentação da informação nutricional contida nas embalagens dos alimentos e observar o impacto na percepção de saudabilidade e na intenção de compra. A pesquisa revelou que os produtos que apresentavam na embalagem mais in- 
formações nutricionais eram considerados produtos mais confiáveis, com maior credibilidade, sendo, então, uma boa escolha na hora da compra.

Após estudo, das 59 mulheres entrevistadas, $61 \%(n=36)$ relataram ler o rótulo dos alimentos contido nas embalagens. Destas, 33\% $(n=12)$ apontaram ter dificuldade para enxergar as informações da rotulagem, em razão da fonte ser pequena ou até mesmo pela cor das letras em relação à cor da embalagem. Ainda $42 \%$ ( $n=15)$ declararam que o mais difícil seria compreender o conteúdo. Com relação às mulheres que não têm o hábito de ler o rótulo, estas somaram $39 \%(n=23)$ e as mesmas revelaram motivos como: não achar importante, ir com um tempo curto ao mercado, não poder parar para observar o rótulo e também por ter o hábito de comprar sempre os mesmos alimentos e já "conhecer" o produto.

Abreu et al. (2015) avaliaram hábitos de compras de 130 consumidores de três supermercados localizados em cidades mineiras e observaram que $85,4 \%$ dos consumidores mencionaram ler os rótulos dos alimentos, o que, segundo eles, é um hábito que faz diferença na hora da escolha de um produto. Outro aspecto importante mencionado foi a qualidade nutricional do alimento, presente em $46,9 \%$ das respostas.

Destaca-se que das 36 entrevistadas que afirmaram ter o hábito de ler as informações que constam nas embalagens dos alimentos, $80 \%(n=29)$ tinham a preocupação voltada para a data de validade do alimento, seguido da lista de ingredientes, com $17 \%(n=6), e$, em último lugar, e menos lembrado pelas mulheres, a Informação Nutricional Complementar (INC), com apenas $3 \%(n=1)$.

Já em relação à composição nutricional dos alimentos, observou-se que o valor calórico é o item mais importante para $25 \%(n=9)$ das mulheres, seguido do açúcar, com $16 \%(n=6)$, e gorduras totais, saturada e o sódio, com $14 \%(n=5)$ cada; os demais nutrientes de declaração obrigatória foram menos lembrados. Acredita-se que este achado tenha relação com o gênero das entrevistadas e as doenças crônicas mais citadas, sendo elas a hipertensão arterial sistêmica e a diabetes mellitus.

É importante ressaltar que a rotulagem dos alimentos possui um papel importantíssimo na educação alimentar e nutricional do consumidor. É por meio da leitura do rótulo que ele se torna capaz de escolher o produto adequado a sua realidade. Nesse processo, cabe ao nutricionista estimular o hábito da leitura, bem como da atenção às orientações nutricionais corretas, pois, desta forma, os indivíduos serão capazes de selecionar os alimentos que mais bem atendem suas necessidades metabólicas e fisiológicas (GARCIA; DE CARVALHO, 2011 ).

As INCs também são formas de informar o consumidor, desde que apresentadas de forma correta. Segundo a Anvisa, é considerada INC qualquer representação que afirme, sugira ou implique que um alimento possui propriedades nutricionais particulares, especialmente, mas não somente, em relação ao seu valor energético e/ou ao seu conteúdo de proteínas, gorduras, carboidratos e fibra alimentar, assim como ao seu conteúdo de vitaminas e minerais (BRASIL 2015). O percentual de observação das INCs no presente estudo foi inexpressivo.

O Quadro 3 aponta a tendência de consumo de alimentos industrializados considerando a redução ou adição de nutrientes para tornar o alimento ou produto alimentício mais "saudável"; normalmente estas características são retratadas na embalagem em forma de INC.

Quadro 2 - Tendência de consumo de alimentos industrializados por mulheres. Itajaí, setembro/2017 a março/2018

\begin{tabular}{|l|c|c|c|c|}
\hline \multirow{2}{*}{ Item } & \multicolumn{2}{|c|}{ Sim } & \multicolumn{2}{|c|}{ Não } \\
\cline { 2 - 5 } & $\mathbf{n}$ & $\mathbf{\%}$ & $\mathbf{n}$ & $\mathbf{\%}$ \\
\hline $\begin{array}{l}\text { Você aceitaria pagar mais por um } \\
\text { alimento com menos GORDURA }\end{array}$ & 49 & 83 & 10 & 17 \\
\hline $\begin{array}{l}\text { Você aceitaria pagar mais por um } \\
\text { alimento com menos SAL }\end{array}$ & 52 & 88 & 07 & 12 \\
\hline $\begin{array}{l}\text { Você aceitaria pagar mais por } \\
\text { um alimento com menos ou sem } \\
\text { AÇÚCAR }\end{array}$ & 45 & 76 & 14 & 24 \\
\hline $\begin{array}{l}\text { Você aceitaria pagar mais por um } \\
\text { alimento com mais FIBRA }\end{array}$ & 48 & 81 & 11 & 19 \\
\hline $\begin{array}{l}\text { Você aceitaria pagar mais por um } \\
\text { alimento com mais VITAMINAS E } \\
\text { MINERAIS }\end{array}$ & 52 & 88 & 7 & 12 \\
\hline $\begin{array}{l}\text { Você aceitaria pagar mais por um } \\
\text { alimento com ADOÇANTE }\end{array}$ & 16 & 27 & 43 & 73 \\
\hline
\end{tabular}

Fonte: Os autores, 2018.

Os resultados do Quadro 2 leva a traçar-se um paralelo com as DCNTs mais presentes, uma vez que a maioria das entrevistadas referiram que aceitariam pagar mais por um alimento com menos sódio, seguido da diminuição de gordura e açúcar respectivamente. Sabe-se, entretanto, que o fato de o alimento ter maior ou menor quantidade de um determinado nutriente não garante sua saudabilidade, uma vez que uma alimentação saudável vai muito além da ingestão de nutrientes envolvendo múltiplos contextos. Destaca-se que, segundo o Guia Alimentar para a População Brasileira (BRASIL, 2014b), a base da nossa alimen- 
tação deveria ser de alimentos in natura ou minimamente processados, correspondentes a alimentos com o mínimo possível de aditivos ou conservantes.

Estes resultados corroboram as ideias de Viana et al. (2017), demonstrando que a preocupação com a alimentação é um hábito constante em diferentes segmentos da população. Para os autores, este fenômeno pode estar associado à acentuada racionalidade em torno do que comer: a imensa oferta de alimentos industrializados, a grande complexidade dos rótulos de alimentos, as políticas públicas que tomam a concepção de alimentação saudável como um dos meios de promoção da qualidade de vida, a galopante inovação na criação de sistemas alimentares cada vez mais sofisticados, crescentes informações em torno da funcionalidade dos alimentos e o que tudo isso proporciona: o incomensurável acervo de informações sobre o assunto.

\section{CONSIDERAÇÕES FINAIS}

A partir dos resultados apresentados foi possível observar que a maioria das mulheres entrevistadas eram as que faziam as compras dos alimentos, em sua maioria adultas de meia idade, com Ensino Fundamental incompleto e renda per capita inferior a um salário mínimo, tendo como doença crônica mais relatada a hipertensão arterial sistêmica, seguida de diabetes mellitus. Verificou-se, ainda, que o fator que mais influência na compra dos alimentos é o preço e que a maioria das mulheres tem um valor máximo para gastar no supermercado por mês e usa lista a fim de gastar menos.

Outra evidência que confirma o preço como fator decisivo na hora da aquisição foi o fato de o alimento estar em promoção. Por outro lado, percebeu-se uma tendência de preocupação com a saudabilidade do produto, associada ao pouco conhecimento do mesmo, posto que a maioria não tinha o hábito de fazer a leitura dos rótulos.

Diante deste contexto, é possível verificar a necessidade de atividades de educação alimentar e nutricional que levem à reflexão sobre o ato de se alimentar, buscando a desassociação entre alimento industrializado e preço com alimentos saudáveis. Neste sentido, cabe aos profissionais inseridos nas Unidades de Saúde esclarecer as vantagens do consumo de alimentos in natura e minimamente processados em relação aos demais, bem como trabalhar informações simples, como a lista de ingredientes dos alimentos processados. Entende-se que, para tanto, faz-se ne- cessário que toda a equipe de saúde das Unidades tenha conhecimento sobre o Guia Alimentar para a População Brasileira (GAPB).

\section{REFERÊNCIAS}

ABREU PINHEIRO, Flávia et al. Perfil de consumidores em relação à qualidade de alimentos e hábitos de compras. Journal of Health Sciences, v. 13, n. 2, 2015.

ANDRADE, Silvânia Suely de Araújo et al. Prevalência de hipertensão arterial autorreferida na população brasileira: análise da Pesquisa Nacional de Saúde, 2013. Epidemiologia e Serviços de Saúde, v. 24, n. 2, p. 297-304, 2015.

BENDINO, Nívea I.; POPOLIM, Welliton D.; OLIVEIRA, C. R. A. Avaliação do conhecimento e dificuldades de consumidores frequentadores de supermercado convencional em relação à rotulagem de alimentos e informação nutricional. Journal of the Health Sciences Institute, v. 30, n. 3, p. 261-265, 2012. BIELEMANN, Renata M. et al. Consumo de alimentos ultraprocessados e seu impacto na dieta de adultos jovens. Rev. Saúde Pública, São Paulo, v. 49, n. 28, 2015.

BORGES, Camila Aparecida et al. Quanto custa para as famílias de baixa renda obterem uma dieta saudável no Brasil? Cadernos de Saúde Pública, v. 31, p. 137-148, 2015.

BRASIL. Organização Pan-Americana da Saúde/Opas. Doenças crônicas não transmissíveis causam 16 milhões de mortes prematuras todos os anos. Brasil, 23 jan. 2015.

BRASIL. Portal da Saúde. Ministério da saúde lança guia alimentar para população brasileira. Brasília-DF, 7 nov. 2014b. BRASIL. Portal da Saúde. Vigilância das doenças crônicas não transmissíveis. Brasília-DF, 4 ago. 2014a.

CARRAZEDO MARQUES DA COSTA FILHO, Murilo; DE MENDONÇA MOTTA, Paulo Cesar. Gestão de orçamento nas compras de supermercado da nova classe média. Revista Pensamento Contemporâneo em Administração, v. 9, n. 4, 2015.

DUNCAN, Bruce Bartholow et al. Doenças crônicas não transmissíveis no Brasil: prioridade para enfrentamento e investigação. Revista de Saúde Pública, São Paulo. v. 46, n. 1, p. 126-134, dez. 2012.

DURANTE, Gabriela Dalcin et al. Diferenças no consumo de alimentos entre homens e mulheres entrevistados pelo inquérito telefônico Vigitel. Revista Brasileira em Promoção da Saúde, v. 30, n. 3, 2017.

FIGUEIRA, Taís Rocha; LOPES, Aline Cristine Souza; MODENA, Celina Maria. Barreiras e fatores promotores do consumo de frutas e hortaliças entre usuários do Programa Academia da Saúde. Revista de Nutrição, v. 29, n. 1, p. 85-95, fev. 2016.

GARCIA, Paloma Popov Custódio; DE CARVALHO, Leiliane Pereira da Silva. Análise da rotulagem nutricional de alimentos diet e light. Ensaios e Ciência: C. Biológicas, Agrárias e da Saúde, v. 15, n. 4, 2011.

GUIBU, lone Aquemi et al. Características principais dos usuários dos serviços de atenção primária à saúde no Brasil. Rev. Saúde Pública, v. 51, n. suppl. 2, 2017. 
HALLES, Claudia Regina; SOKOLOWSKI, Rivelto; HILGEMBERG, Emerson Martins. O planejamento financeiro como instrumento de qualidade de vida. SEMINÁRIO DE POLÍTICAS PÚBLICAS NO PARANÁ: ESCOLA DO GOVERNO E UNIVERSIDADES ESTADUAIS, 1., 2011. Curitiba, 2011.

LINDEMANN, Ivana Loraine; OLIVEIRA, Riceli Rodeghiero; MENDOZA-SASSI, Raúl Andres. Dificuldades para alimentação saudável entre usuários da atenção básica em saúde e fatores associados. Ciência \& Saúde Coletiva, Pelotas, v. 21, n. 2, p. 599-610, fev. 2016.

MALTA, Deborah Carvalho et al. Mortalidade por doenças crônicas não transmissíveis no Brasil e suas regiões, 2000 a 2011. Epidemiologia e Serviços de Saúde, v. 23, n. 4, p. 599608, 2014.

MARTINS, Ana Paula Bortoletto et al. Participação crescente de produtos ultraprocessados na dieta brasileira (19872009). Revista de Saúde Pública, v. 47, n. 4, p. 656-665, 2013.

MONTEIRO, C. A. et al. A new classification of foods based on the extent and purpose of their processing. Cad. Saúde Pública, v. 26, n. 11, p. 49, 2010.
MONTEIRO, C. A. et al. Increasing consumption of ultra-processed foods and likely impact on human health: evidence from Brazil. Public Health Nutr, v. 14, n. 1, p. 5-13, 2011.

OLIVEIRA, Tatiana Coura et al. Concepções sobre práticas alimentares em mulheres de camadas populares no Rio de Janeiro, RJ, Brasil: transformações e ressignificações. Interface - Comunicação, Saúde, Educação, v. 22, n. 65, p. 435446, 19 out. 2017.

RICCI, Bianca Castro da Silva Maraninchi. A forma de apresentação das informações nutricionais em embalagens de alimentos e o impacto na decisão de compra dos pais. 2016. 152f. Dissertação (Mestrado em Administração) - Faculdade de Administração, Contabilidade e Economia, Pontifícia Universidade Católica, Porto Alegre, Rio Grande Sul, 2016.

SANTOS, Cláudia Maria Barbosa et al. Experiência de extensão: "Rotulagem nutricional: conheça o que você consome". Revista Ciência em Extensão, v. 12, n. 4, p. 160-173, 2016.

VIANA, Marcia Regina et al. A racionalidade nutricional e sua influência na medicalização da comida no Brasil. Ciência \& Saúde Coletiva, v. 22, p. 447-456, 2017. 